\title{
Unsupervised Induction of Stochastic Context-Free Grammars using Distributional Clustering
}

\author{
Alexander Clark \\ Cognitive and Computing Sciences, \\ University of Sussex, \\ Brighton BN1 9QH, \\ United Kingdom \\ alexclcogs.susx.ac.uk
}

\begin{abstract}
An algorithm is presented for learning a phrase-structure grammar from tagged text. It clusters sequences of tags together based on local distributional information, and selects clusters that satisfy a novel mutual information criterion. This criterion is shown to be related to the entropy of a random variable associated with the tree structures, and it is demonstrated that it selects linguistically plausible constituents. This is incorporated in a Minimum Description Length algorithm. The evaluation of unsupervised models is discussed, and results are presented when the algorithm has been trained on 12 million words of the British National Corpus.
\end{abstract}

\section{Introduction}

In this paper I present an algorithm using context distribution clustering (CDC) for the unsupervised induction of stochastic context-free grammars (SCFGs) from tagged text. Previous research on completely unsupervised learning has produced poor results, and as a result researchers have resorted to mild forms of supervision. Magerman and Marcus(1990) use a distituent grammar to eliminate undesirable rules. Pereira and Schabes(1992) use partially bracketed corpora and Carroll and Charniak(1992) restrict the set of non-terminals that may appear on the right hand side of rules with a given left hand side. The work of van Zaanen (2000) does not have this problem, and appears to perform well on small data sets, but it is not clear whether it will scale up to large data sets. Adriaans et al. (2000)

\author{
ISSCO / ETI, \\ University of Geneva, \\ UNI-MAIL, Boulevard du Pont-d' Arve, 40 \\ CH-1211 Genève 4, \\ Switzerland
}

presents another algorithm but its performance on authentic natural language data appears to be very limited.

The work presented here can be seen as one more attempt to implement Zellig Harris's distributional analysis (Harris, 1954), the first such attempt being (Lamb, 1961).

The rest of the paper is arranged as follows: Section 2 introduces the technique of distributional clustering and presents the results of a preliminary experiment. Section 3 discusses the use of a novel mutual information (MI) criterion for filtering out spurious candidate non-terminals. Section 4 shows how this criterion is related to the entropy of a certain random variable, and Section 5 establishes that it does in fact have the desired effect. This is then incorporated in a Minimum Description Length (MDL) algorithm outlined in Section 6. I discuss the difficulty of evaluating this sort of unsupervised algorithm in Section 7, and present the results of the algorithm on the British National Corpus (BNC). The paper then concludes after a discussion of avenues for future research in Section 8.

\section{Distributional clustering}

Distributional clustering has been used in many applications at the word level, but as has been noticed before (Finch et al., 1995), it can also be applied to the induction of grammars. Sets of tag sequences can be clustered together based on the contexts they appear in. In the work here I consider the context to consist of the part of speech tag immediately preceding the sequence and the tag immediately following it. The dependency between these is critical, as we shall see, so the context distribution therefore has $k^{2}$ parameters, where $k$ is the number of tags, rather than 


\begin{tabular}{|c|c|c|c|c|c|c|}
\hline $\mathrm{ATO}$ & AJO & NNO & & $\mathrm{AJO}$ & $\mathrm{AJC}$ & \\
\hline ATO & AJO & NN1 & & AJO & $\mathrm{CJC}$ & AJO \\
\hline ATO & AJ0 & NN2 & & AV0 & $\mathrm{AJC}$ & \\
\hline $\mathrm{ATO}$ & AV0 & AJ0 & NN1 & AV0 & AVC & AJO \\
\hline ATO & NNO & & & ORD & & \\
\hline ATO & NN1 & PRP & AT0 NN1 & & & \\
\hline ATO & NN1 & & & & & \\
\hline
\end{tabular}

Table 1: Some of the more frequent sequences in two good clusters

the $2 k$ parameters it would have under an independence assumption. The context distribution can be thought of as a distribution over a twodimensional matrix.

The data set for all the results in this paper consisted of 12 million words of the British National Corpus, tagged according to the CLAWS-5 tag set, with punctuation removed.

There are 76 tags; I introduced an additional tag to mark sentence boundaries. I operate exclusively with tags, ignoring the actual words. My initial experiment clustered all of the tag sequences in the corpus that occurred more than 5000 times, of which there were 753, using the $k$ means algorithm with the $L_{1}$-norm or city-block metric applied to the context distributions. Thus sequences of tags will end up in the same cluster if their context distributions are similar; that is to say if they appear predominantly in similar contexts. I chose the cutoff of 5000 counts to be of the same order as the number of parameters of the distribution, and chose the number of clusters to be 100 .

To identify the frequent sequences, and to calculate their distributions I used the standard technique of suffix arrays (Gusfield, 1997), which allows rapid location of all occurrences of a desired substring.

As expected, the results of the clustering showed clear clusters corresponding to syntactic constituents, two of which are shown in Table 1. Of course, since we are clustering all of the frequent sequences in the corpus we will also have clusters corresponding to parts of constituents, as can be seen in Table 2. We obviously would not want to hypothesise these as constituents: we therefore need some criterion for filtering out these spurious candidates.

\begin{tabular}{|llll|lll|}
\hline AJ0 & NN1 & AT0 & & CJC & AT0 & AJ0 \\
AJ0 & NN1 & PRF & AT0 & CJC & AT0 & \\
AJ0 & NN1 & PRP & AT0 & CJC & CRD & \\
NN1 & AT0 & AJ0 & & CJC & DPS & \\
NN1 & AT0 & & & CJC & PRP & AT0 \\
NN1 & CJC & AJ0 & & PRF & AJ0 & \\
\hline
\end{tabular}

Table 2: Some of the sequencess in two bad clusters

\section{Mutual Information}

The criterion I propose is that with real constituents, there is high mutual information between the symbol occurring before the putative constituent and the symbol after-i.e. they are not independent. Note that this is unrelated to Magerman and Marcus's MI criterion which is the (generalised) mutual information of the sequence of symbols itself. I will justify this in three ways intuitively, mathematically and empirically.

Intuitively, a true constituent like a noun phrase can appear in a number of different contexts. This is one of the traditional constituent tests. A noun phrase, for example, appears frequently either as the subject or the object of a sentence. If it appears at the beginning of a sentence it is accordingly quite likely to be followed by a finite verb. If on the other hand it appears after the finite verb, it is more likely to be followed by the end of the sentence or a preposition. A spurious constituent like PRP ATO will be followed by an N-bar regardless of where it occurs. There is therefore no relation between what happens immediatly before it, and what happens immediately after it. Thus there will be a higher dependence or correlation with the true constituent than with the erroneous one.

\section{Mathematical Justification}

We can gain some insight into the significance of the MI criterion by analysing it within the framework of SCFGs. We are interested in looking at the properties of the two-dimensional distributions of each non-terminal. The terminals are the part of speech tags of which there are $T$. For each terminal or non-terminal symbol $X$ we define four distributions, $L(X), P(X), S(X), R(X)$, over $T$ or equivalently $T$-dimensional vectors. 
Two of these, $P(X)$ and $S(X)$ are just the prefix and suffix probability distributions for the symbol(Stolcke, 1995): the probabilities that the string derived from $X$ begins (or ends) with a particular tag. The other two $L(X), R(X)$ for left distribution and right distribution, are the distributions of the symbols before and after the nonterminal. Clearly if $X$ is a terminal symbol, the strings derived from it are all of length 1 , and thus begin and end with $X$, giving $P(X)$ and $S(X)$ a very simple form.

If we consider each non-terminal $N$ in a SCFG, we can associate with it two random variables which we can call the internal and external variables. The internal random variable is the more familiar and ranges over the set of rules expanding that non-terminal. The external random variable, $Z_{N}$, is defined as the context in which the non-terminal appears. Every non-root occurrence of a non-terminal in a tree will be generated by some rule $r$, that it appears on the right hand side of. We can represent this as $(r, i)$ where $r$ is the rule, and $i$ is the index saying where in the right hand side it occurs. The index is necessary since the same non-terminal symbol might occur more than once on the right hand side of the same rule. So for each $N, Z_{N}$ can take only those values of $(r, i)$ where $N$ is the $i$ th symbol on the right hand side of $r$.

The independence assumptions of the SCFG imply that the internal and external variables are independent, i.e. have zero mutual information. This enables us to decompose the context distribution into a linear combination of the set of marginal distributions we defined earlier.

Let us examine the context distribution of all occurrences of a non-terminal $N$ with a particular value of $Z_{N}$. We can distinguish three situations: the non-terminal could appear at the beginning, middle or end of the right hand side. If it occurs at the beginning of a rule $r$ with left hand side $X$, and the rule is $X \rightarrow N Y \ldots$ then the terminal symbol that appears before $N$ will be distributed exactly according to the symbol that occurs before $X$, i.e. $L(N)=L(X)$. The non-terminal symbol that occurs after $N$ will be distributed according to the symbol that occurs at the beginning of the symbol that occurs after $N$ in the right hand side of the rule, so $R(N)=P(Y)$. By the inde- pendence assumption, the joint distribution is just the product of the two marginals.

$$
D\left(N \mid Z_{N}=(r, 1)\right)=L(X) \times P(Y)
$$

Similarly if it occurs at the end of a rule $X \rightarrow$ $\ldots W N$ we can write it as

$$
D\left(N \mid Z_{N}=(r,|r|)\right)=S(W) \times R(X)
$$

and if it occurs in the middle of a rule $X \rightarrow$ $\ldots W N Y \ldots$ we can write it as

$$
D\left(N \mid Z_{N}=(r, i)\right)=S(W) \times P(Y)
$$

The total distribution of $N$ will be the normalised expectation of these three with respect to $P\left(Z_{N}\right)$. Each of these distributions will have zero mutual information, and the mutual information of the linear combination will be less than or equal to the entropy of the variable combining them, $H\left(Z_{N}\right)$.

In particular if we have

$$
P(X=x, Y=y)=\sum_{i} \alpha_{i} p_{i}(x) q_{i}(y)
$$

using Jensen's inequality we can prove that

$$
I(X ; Y) \leq \sum-\alpha_{i} \log \alpha_{i}
$$

We will have equality when the context distributions are sufficiently distinct. Therefore

$$
M I(N) \leq H\left(Z_{N}\right)
$$

Thus a non-terminal that appears always in the same position on the right hand side of a particular rule, will have zero MI, whereas a nonterminal that appears on the right hand side of a variety of different rules will, or rather may, have high MI.

This is of limited direct utility, since we do not know which are the non-terminals and which are other strings, but this establishes some circumstances under which the approach won't work. Some of these are constraints on the form of the grammar, namely that no non-terminal can appear in just a single place on the right hand side of a single rule. Others are more substantive constraints on the sort of languages that can be learned. 


\begin{tabular}{|llll|}
\hline Symbol & Description & Number of rules & Most Frequent \\
\hline \hline NP & Noun Phrase & 107 & AT0 NN1 \\
AVP & Adverb Phrase & 6 & AV0 AV0 \\
PP & Prep. Phrase & 47 & PRP NP \\
S & Clause & 19 & PNP VVD NP \\
XPCONJ & Phrase and Conj. & 5 & PP CJC \\
N-BAR & & 121 & AJ0 NN1 \\
S-SUB & Subordinate Clause $?$ & 58 & S-SUB PP \\
NT-NP0AV0 & & 3 & PNP AV0 \\
NT-VHBVBN & Finite copula phrase & 12 & VM0 VBI \\
NT-AV0AJ0 & Adjective Phrase & 11 & AVO AJ0 \\
NT-AJ0CJC & & 10 & AJ0 CJC \\
NT-PNPVBBVVN & Subject + copula & 21 & PNP VBD \\
\hline
\end{tabular}

Table 3: Non-terminals produced during first 20 iterations of the algorithm.

\section{Experimental Verification}

To implement this, we need some way of deciding a threshhold which will divide the sheep from the goats. A simple fixed threshhold is undesirable for a number of reasons. One problem with the current approach is that the maximum likelihood estimator of the mutual information is biased, and tends to over-estimate the mutual information with sparse data ( $\mathrm{Li}, 1990)$. A second problem is that there is a "natural" amount of mutual information present between any two symbols that are close to each other, that decreases as the symbols get further apart. Figure 1 shows a graph of how the distance between two symbols affects the MI between them. Thus if we have a sequence of length 2 , the symbols before and after it will have a distance of 3 , and we would expect to have a MI of 0.05 . If it has more than this, we might hypothesise it as a constituent; if it has less, we discard it.

In practice we want to measure the MI of the clusters, since we will have many more counts, and that will make the MI estimate more accurate. We therefore compute the weighted average of this expected MI, according to the lengths of all the sequences in the clusters, and use that as the criterion. Table 4 shows how this criterion separates valid from invalid clusters. It eliminated 55 out of 100 clusters

In Table 4, we can verify this empirically: this criterion does in fact filter out the undesirable sequences. Clearly this is a powerful technique for

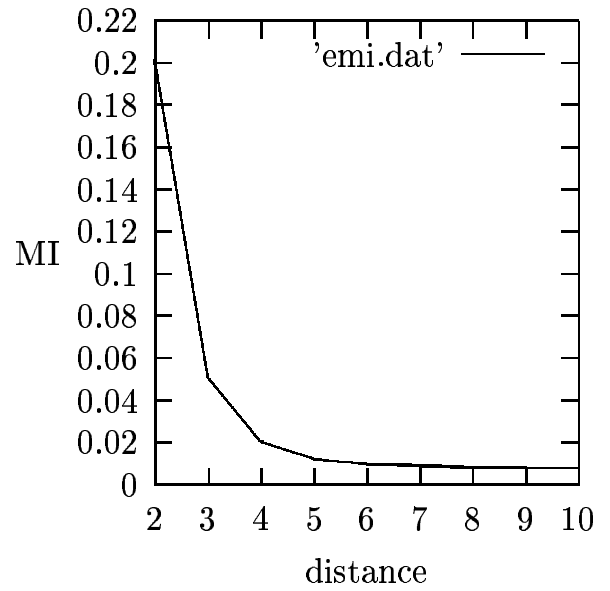

Figure 1: Graph of expected MI against distance.

\begin{tabular}{|lllll|}
\hline \multicolumn{2}{|l}{ Cluster } & Actual MI & Exp. MI & Valid \\
\hline \hline AT0 & NN1 & 0.11 & 0.04 & Yes \\
AT0 NP0 NP0 & 0.13 & 0.02 & Yes \\
PRP AT0 NN1 & 0.06 & 0.02 & Yes \\
AV0 & AJ0 & 0.27 & 0.1 & Yes \\
\hline NN1 AT0 & 0.008 & 0.02 & No \\
AT0 AJ0 & 0.02 & 0.03 & No \\
VBI AT0 & 0.01 & 0.02 & No \\
PRP AT0 & 0.01 & 0.03 & No \\
\hline
\end{tabular}

Table 4: Four valid clusters where the actual MI is greater than the expected MI, and four invalid clusters which fail the test. The four invalid clusters clearly are not constituents according to traditional criteria. 
identifying constituents.

\section{Minimum Description Length}

This technique can be incorporated into a grammar induction algorithm. We use the clustering algorithm to identify sets of sequences that can be derived from a single non-terminal. The MI criterion allows us to find the right places to cut the sentences up; we look for sequences where there are interesting long-range dependencies. Given these potential sequences, we can then hypothesise sets of rules with the same right hand side. This naturally suggests a minimum description length (MDL) or Bayesian approach (Stolcke, 1994; Chen, 1995). Starting with the maximum likelihood grammar, which has one rule for each sentence type in the corpus, and a single nonterminal, at each iteration we cluster all frequent strings, and filter according to the MI criterion discussed above.

We then greedily select the cluster that will give the best immediate reduction in description length, calculated according to a theoretically optimal code. We add a new non-terminal with rules for each sequence in the cluster. If there is a sequence of length 1 with a nonterminal in it, then instead of adding a new nonterminal, we add rules expanding that old nonterminal. Thus, if we have a cluster which consists of the three sequences NP, NP PRP NP and NP PRF NP we would merely add the two rules $\mathrm{NP} \rightarrow \mathrm{NP} \quad \mathrm{PRP} \quad \mathrm{NP}$ and $\mathrm{NP} \rightarrow \mathrm{NP} \quad \mathrm{PRF} \quad \mathrm{NP}$, rather than three rules with a new non-terminal on the left hand side. This allows the algorithm to learn recursive rules, and thus context-free grammars.

We then perform a partial parse of all the sentences in the corpus, and for each sentence select the path through the chart that provides the shortest description length, using standard dynamic programming techniques. This greedy algorithm is not ideal, but appears to be unavoidable given the computational complexity. Following this, we aggregate rules with the same right hand sides and repeat the operation.

Since the algorithm only considers strings whose frequency is above a fixed threshhold, the application of a rule in rewriting the corpus will often result in a large number of strings being rewritten so that they are the same, thus bringing a particular sequence above the threshhold. Then at the next iteration, this sequence will be examined by the algorithm. Thus the algorithm progressively probes deeper into the structure of the corpus as syntactic variation is removed by the partial parse of low level constituents.

Singleton rules require special treatment; I have experimented with various different options, without finding an ideal solution. The results presented here use singleton rules, but they are only applied when the result is necessary for the application of a further rule. This is a natural consequence of the shortest description length choice for the partial parse: using a singleton rule increases the description length.

The MDL gain is very closely related to the mutual information of the sequence itself under standard assumptions about optimal codes (Cover and Thomas, 1991). Suppose we have two symbols $x$ and $y$ that occur $n_{x}$ and $n_{y}$ times in a corpus of length $N$ and that the sequence $x y$ occurs $n_{x y}$ times. We could instead create a new symbol that represents $x y$, and rewrite the corpus using this abbreviation. Since we would use it $n_{x y}$ times, each symbol would require $\log N / n_{x y}$ nats. The symbols $x$ and $y$ have codelengths of $\log N / n_{x}$ and $\log N / n_{y}$, so for each pair $x y$ that we rewrite, under reasonable approximations, we have a reduction in code length of

$$
\begin{aligned}
\Delta L & \approx-\log N / n_{x y}+\log N / n_{x}+\log N / n_{y} \\
& \approx \log \frac{p(x y)}{p(x) p(y)}
\end{aligned}
$$

which is the point-wise mutual information between $x$ and $y$.

I ran the algorithm for 40 iterations. Beyond this point the algorithm appeared to stop producing plausible constituents. Part of the problem is to do with sparseness: it requires a large number of samples of each string to estimate the distributions reliably.

\section{Evaluation}

Evaluation of unsupervised algorithms is difficult. One evaluation scheme that has been used is to compare the constituent structures produced by the grammar induction algorithm against a treebank, and use PARSEVAL scoring metrics, as 
advocated by (van Zaanen and Adriaans, 2001); i.e. use exactly the same evaluation as is used for supervised learning schemes. This proposal fails to take account of the fact that the annotation scheme used in any corpus, does not reflect some theory-independent reality, but is the product of various more or less arbitrary decisions by the annotators (Carroll et al., 1998). Given a particular annotation scheme, the structure in the corpus is not arbitrary, but the choice of annotation scheme inevitably is. Thus expecting an unsupervised algorithm to converge on one particular annotation scheme out of many possible ones seems overly onerous.

It is at this point that one must question what the point of syntactic structure is: it is not an end in itself but a precursor to semantics. We need to have syntactic structure so we can abstract over it when we learn the semantic relationships between words. Seen in this context, the suggestion of evaluation based on dependency relationships amongst words(Carroll et al., 1998) seems eminently sensible.

With unsupervised algorithms, there are two aspects to the evaluation; first how good the annotation scheme is, and secondly how good the parsing algorithm is - i.e. how accurately the algorithm assigns the structures. Since we have a very basic non-lexicalised parser, I shall focus on evaluating the sort of structures that are produced, rather than trying to evaluate how well the parser works. To facilitate comparison with other techniques, I shall also present an evaluation on the ATIS corpus.

Pereira and Schabes (1992) establish that evaluation according to the bracketing accuracy and evaluation according to perplexity or crossentropy are very different. In fact, the model trained on the bracketed corpus, although scoring much better on bracketing accuracy, had a higher (worse) perplexity than the one trained on the raw data. This means that optimising the likelihood of the model may not lead you to a linguistically plausible grammar.

In Table 3 I show the non-terminals produced during the first 20 iterations of the algorithm. Note that there are less than 20 of them, since as mentioned above sometimes we will add more rules to an existing non-terminal. I have taken the

\begin{tabular}{|ll|}
\hline Count & Right Hand Side \\
\hline \hline 255793 & AT0 NN1 \\
104314 & NP PP \\
103727 & AT0 AJ0 NN1 \\
73151 & AT0 NN2 \\
72686 & DPS NN1 \\
52202 & AJ0 NN2 \\
51575 & DT0 NN1 \\
35473 & NP NP \\
34523 & DT0 NN2 \\
34140 & AV0 NP \\
\hline
\end{tabular}

Table 5: Ten most frequent rules expanding NP. Note that three of them are recursive.

liberty of attaching labels such as NP to the nonterminals where this is well justified. Where it is not, I leave the symbol produced by the program which starts with NT-. Table 5 shows the most frequent rules expanding the NP non-terminal. Note that there is a good match between these rules and the traditional phrase structure rules.

To facilitate comparison with other unsupervised approaches, I performed an evaluation against the ATIS corpus. I tagged the ATIS corpus with the CLAWS tags used here, using the CLAWS demo tagger available on the web, removed empty constituents, and adjusted a few tokenisation differences (at least is one token in the BNC.) I then corrected a few systematic tagging errors. This might be slightly controversial. For example, "Washington D C" which is three tokens was tagged as NPO ZZO ZZO where ZZO is a tag for alphabetic symbols. I changed the $z z 0$ tags to NP 0. In the BNC, that I trained the model on, the DC is a single token tagged as NP 0 , and in the ATIS corpus it is marked up as a sequence of three NNP. I did not alter the mark up of flight codes and so on that occur frequently in this corpus and very infrequently in the BNC.

It is worth pointing out that the ATIS corpus is a very simple corpus, of radically different structure and markup to the BNC. It consists primarily of short questions and imperatives, and many sequences of letters and numbers such as T W A, A P 57 and so on.

For instance, a simple sentence like "Show me the meal" has the gold standard parse:

(S (VP (VB Show) 


\section{(NP (PRP me)) \\ (NP (DT the) \\ (NN meal))) )}

and is parsed by this algorithm as

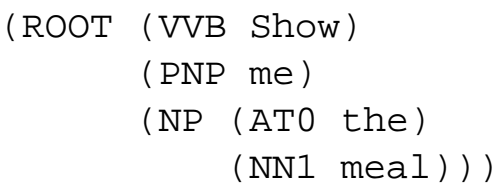

According to this evaluation scheme its recall is only $33 \%$, because of the presence of the nonbranching rules, though intuitively it has correctly identified the bracketing. However, the crossing brackets measures overvalues these algorithms, since they produces only partial parses - for some sentences my algorithm produces a completely flat parse tree which of course has no crossing brackets.

I then performed a partial parse of this data using the SCFG trained on the BNC, and evaluated the results against the gold-standard ATIS parse using the PARSEVAL metrics calculated by the EVALB program. Table 6 presents the results of the evaluation on the ATIS corpus, with the results on this algorithm (CDC) compared against two other algorithms, EMILE (Adriaans et al., 2000) and ABL (van Zaanen, 2000). The comparison presented here allows only tentative conclusions for these reasons: first, there are minor differences in the test sets used; secondly, the CDC algorithm is not completely unsupervised at the moment as it runs on tagged text, whereas ABL and EMILE run on raw text, though since the ATIS corpus has very little lexical ambiguity the difference is probably quite minor; thirdly, it is worth reiterating that the $\mathrm{CDC}$ algorithm was trained on a radically different and much more complex data set. However, we can conclude that the CDC algorithm compares favourably to other unsupervised algorithms.

\section{Future Work}

Preliminary experiments with tags derived automatically using distributional clustering (Clark, 2000), have shown essentially the same results. It appears that for the simple constituents that are being constructed in the work presented here, they are sufficiently accurate. This makes the algorithm completely unsupervised.
I have so far used the simplest possible metric and clustering algorithm; there are much more sophisticated hierarchical clustering algorithms that might perform better. In addition, I will explore the use of a lexicalised formalism.

This algorithm uses exclusively bottom-up information; the standard estimation and parsing algorithms use the interaction between bottom-up and top-down information, or inside and outside probabilities to direct the search. It should be possible to add this to the algorithm, though a full inside-outside re-estimation is not computationally feasible at the moment.

The greediness of the algorithm causes many problems. In particular, it makes the algorithm very sensitive to the order in which the rules are acquired. If a rule that rewrites ATO NN1 is applied before a noun noun compounding rule then we will end up with lots of sequences of NP NN1 that will inevitably lead to a rule of the form NP $\rightarrow$ NP NN1. There are possibilities of modifications that would allow the algorithm to delay committing unambiguously to a particular analysis.

\section{Conclusion}

In conclusion, distributional clustering can form the basis of a grammar induction algorithm, by hypothesising sets of rules expanding the same non-terminal. The mutual information criterion proposed here can filter out spurious constituents. The particular algorithm presented here is rather crude, but serves to illustrate the effectiveness of the general technique. The algorithm is computationally expensive, and requires large amounts of memory to run efficiently. Though the results presented here are preliminary, I have shown how an unsupervised grammar induction algorithm can induce at least part of a linguistically plausible grammar from a large mixed corpus of natural language.

\section{Acknowledgements}

This work was performed as part of the European TMR network Learning Computational Grammars. I am grateful to Bill Keller, Susan Armstrong, Andrei Popescu-Belis and an anonymous reviewer for helpful comments. 


\begin{tabular}{|ll|lll|lll|}
\hline Algorithm & Iterations & UR & UP & F-score & CB & 0 CB & $\leq 2$ CB \\
\hline \hline EMILE & & 16.8 & 51.6 & 25.4 & 0.84 & 47.4 & $\mathbf{9 3 . 4}$ \\
ABL & & $\mathbf{3 5 . 6}$ & 43.6 & 39.2 & 2.12 & 29.1 & 65.0 \\
\hline \hline CDC & 10 & 23.7 & 57.2 & 33.5 & $\mathbf{0 . 8 2}$ & $\mathbf{5 7 . 3}$ & 90.9 \\
CDC & 20 & 27.9 & 54.2 & 36.8 & 1.10 & 54.9 & 85.0 \\
CDC & 30 & 33.3 & $\mathbf{5 4 . 9}$ & 41.4 & 1.31 & 48.3 & 80.5 \\
CDC & 40 & 34.6 & 53.4 & $\mathbf{4 2 . 0}$ & 1.46 & 45.3 & 78.2 \\
\hline
\end{tabular}

Table 6: Results of evaluation on ATIS corpus. UR is unlabelled recall, UP is unlabelled precision, CB is average number of crossing brackets, $\leq 2 \mathrm{CB}$ is percentage with two or fewer crossing brackets. The results for EMILE and ABL are taken from (van Zaanen and Adriaans, 2001)

\section{References}

Pieter Adriaans, Marten Trautwein, and Marco Vervoort. 2000. Towards high speed grammar induction on large text corpora. In Vaclav Hlavac, Keith G. Jeffery, and Jiri Wiedermann, editors, SOFSEM 2000: Theory and Practice of Informatics, pages 173-186. Springer Verlag.

Glenn Carroll and Eugene Charniak. 1992. Two experiments on learning probabilistic dependency grammars from corpora. Technical Report CS-9216, Department of Computer Science, Brown University, March.

John Carroll, Ted Briscoe, and Antonio Sanfilippo. 1998. Parser evaluation: a survey and a new proposal. In Proceedings of the 1st International Conference on Language Resources and Evaluation, pages 447-454, Granada, Spain.

Stanley Chen. 1995. Bayesian grammar induction for language modelling. In Proceedings of the 33rd Annual Meeting of the ACL, pages 228-235.

Alexander Clark. 2000. Inducing syntactic categories by context distribution clustering. In Proceedings of CoNLL-2000 and LLL-2000, pages 91-94, Lisbon, Portugal.

Thomas M. Cover and Joy A. Thomas. 1991. Elements of Information Theory. Wiley Series in Telecommunications. John Wiley \& Sons.

S. Finch, N. Chater, and M. Redington. 1995. Acquiring syntactic information from distributional statistics. In Joseph P. Levy, Dimitrios Bairaktaris, John A. Bullinaria, and Paul Cairns, editors, Connectionist Models of Memory and Language. UCL Press.

Dan Gusfield. 1997. Algorithms on Strings, Trees and Sequences: Computer Science and Computational Biology. Cambridge University Press.

Zellig Harris. 1954. Distributional structure. In J. A. Fodor and J. J. Katz, editors, The Structure of Language, pages 33-49. Prentice-Hall.
Sydney M. Lamb. 1961. On the mechanisation of syntactic analysis. In 1961 Conference on $\mathrm{Ma}$ chine Translation of Languages and Applied Language Analysis, volume 2 of National Physical Laboratory Symposium No. 13, pages 674-685. Her Majesty's Stationery Office, London.

W. Li. 1990. Mutual information functions versus correlation functions. Journal of Statistical Physics, 60:823-837.

David M. Magerman and Mitchell P. Marcus. 1990. Parsing a natural language using mutual information statistics. In Proceedings of the Eighth $\mathrm{Na}$ tional Conference on Artificial Intelligence, August.

Fernando Pereira and Yves Schabes. 1992. Insideoutside reestimation from partially bracketed corpora. In Proceedings of ACL '92, pages 128-135.

Andreas Stolcke. 1994. Bayesian Learning of Probabilistic Language Models. Ph.D. thesis, Dept. of Electrical Engineering and Computer Science, University of California at Berkeley.

Andreas Stolcke. 1995. An efficient probabilistic context-free parsing algorithm that computes prefix probabilities. Computational Linguistics, 21(2):165-202, June.

Menno van Zaanen and Pieter Adriaans. 2001. Comparing two unsupervised grammar induction systems: Alignment-based learning vs. emile. Research Report Series 2001.05, School of Computing, University of Leeds, March.

Menno van Zaanen. 2000. ABL: Alignment-based learning. In COLING 2000 - Proceedings of the 18th International Conference on Computational Linguistics. 